\title{
ON A THEOREM OF RUDIN AND KLEE
}

\section{ERNEST MICHAEL ${ }^{1}$}

Let $X$ and $Y$ be separable metric spaces, and $C(X, Y)$ the space of continuous functions from $X$ to $Y$. In [2], M. E. Rudin and V. L. Klee proved that $C(X, Y)$ is hereditarily Lindelö in the pointwise topology. ${ }^{2}$ Using an idea obtained in conversation from Professor S. Mazur, we can actually show

THEOREM 1. There exists on $C(X, Y)$ a topology I with countable base which makes the map $(f, x) \rightarrow f(x)$ jointly continuous. ${ }^{3}$ Hence $\Im$ is finer than the compact-open topology, which is therefore-along with the pointwise topology-both hereditarily Lindelöf and hereditarily separable.

PRoof. Let $u$ and $v$ be countable bases for $X$ and $Y$, respectively, and let $J$ have as sub-base the collection of sets $\{f \in C(X, Y) \mid f(U) \subset V\}$, with $U \in \mathcal{U}$ and $V \in \mathcal{V}$. Then $\Im$ certainly has a countable base. Clearly $\Im$ also makes $(f, x) \rightarrow f(x)$ jointly continuous, and hence $[1$, p. 223] is finer than the compact-open topology. Finally, every subset of $C(X, Y)$ is certainly Lindelöf and separable for the countable-base topology $\mathfrak{I}$, and hence also for any coarser topology.

\section{REFERENCES}

1. J. L. Kelley, General topology, Toronto, Van Nostrand, 1955.

2. M. E. Rudin and V. L. Klee, $A$ note on certain function spaces, Arch. Math. vol. 7 (1956) pp. 469-470.

\section{UNIVERSITY OF WASHINGTON}

Received by the editors October 7, 1960.

${ }^{1}$ Supported by an N.S.F. contract and a Guggenheim Fellowship.

2 The terminology is the same as in J. L. Kelley [1].

3 A metric giving such a topology was described to the author by Professor S. Mazur. The topology $J$ given in our proof is simpler, but in general not metrizable. 